Check for updates

1 London

2 The BMJ

rcoombes@bmj.com

Cite this as: BMJ2020;370:m2670

http://dx.doi.org/10.1136/bmj.m2670

Published: 8 July 2020
COVID-19

\section{Covid-19: The inside story of the RECOVERY trial}

The UK's flagship covid-19 clinical trial has been hailed worldwide-but some say it is far from perfect. Jacqui Wise and Rebecca Coombes unpick the criticisms that still surround a vital cog in the pandemic response

\author{
Jacqui Wise, ${ }^{1}$ Rebecca Coombes ${ }^{2}$
}

On 16 June the world heard that the first proven lifesaving treatment for covid-19 had been found. Dexamethasone, a widely and cheaply available steroid, was reported to have cut deaths by a third among hospital patients with covid-19 who needed ventilation and by a fifth among patients receiving oxygen only. ${ }^{1}$ In the chaotic, fear filled first half of 2020, this was at last an evidence based treatment from a randomised controlled trial, showcasing what the collective strengths of the NHS could achieve.

Conducted by researchers at Oxford University, the ongoing Randomised Evaluation of Covid-19 Therapy (RECOVERY) trial involves all major hospitals in the UK on an unprecedented scale and as many as 3500 doctors, nurses, and research staff, including consultants, junior doctors, and those newly graduated. Within its first three months it reported its first policy changing result: that the widely promoted antimalarial drug hydroxychloroquine was ineffective. This was swiftly followed by the dexamethasone announcement, seen by many as a much needed ray of hope among the gloom of spiralling infections and deaths.

“The UK has really delivered here,” says Martin Landray, deputy chief investigator of RECOVERY. "It involves hospitals from Truro to the Western Isles, Northern Ireland across to King's Lynn. The patients have been fabulous: they were ill, frightened, alone, and elderly. The success is down to amazing teamwork across the clinical community and the incredible support of patients and their families."

But global recognition and headlines also bring intense scrutiny. Alongside international praise RECOVERY has drawn criticisms from scientists about transparency and a worrying trend for announcing trial results by press release and without the underlying data.

\section{Press release or peer review?}

When the dexamethasone result was announced on 16 June it came unexpectedly early and not in a research paper but a media announcement. The UK government told NHS hospitals to act immediately, even though at that point the data had not yet been published in full as either a preprint or in a peer reviewed journal; it was a week later that a preprint appeared on the medRxiv preprint server.

Scientists are worried about the lack of public scrutiny of the data before major policy decisions were made, particularly given the retractions of high profile papers in journals such as the Lancet and New England Journal of Medicine just weeks earlier. ${ }^{2}$ The arm of RECOVERY evaluating hydroxychloroquine was ended earlier in June after the Medicines and Healthcare Products Agency (MHRA) asked the investigators to review the data early, prompted by the Lancet retraction. Their analysis concluded that the drug conferred no meaningful mortality benefit in the treatment of covid-19, leading to the trial's first announcement of results, although again this was done through the media. At the time of writing the full data have still not been published. ${ }^{3}$

"I think it is irresponsible to release the results only in a press release; a press release is not evidence," says Tobias Kurth, professor of epidemiology and public health at the Charité Berlin University of Medicine and one of The BMJ's statistical advisers. "This habit has to stop now. Even though we are in a difficult situation and urgently need to find something that works, it is important to show all the methods and data."

Landray's defence is that the results were so stark that they had to be publicised, especially in the case of dexamethasone, as it was the first evidence of a treatment that reduces mortality in patients with

What happened between the subgroups in the dexamethasone trial was very unusual, he says. "There was a very clear benefit, especially for patients on ventilators. This was a pre-specified analysis with a highly statistically significant difference between the patients depending on the level of respiratory support they were receiving at the start of the study. Importantly, we saw no benefit (and the possibility of harm) among patients not requiring any form of respiratory support.”

"If you look at Brazil, Mexico, or India, with thousands of patients dying every week, there will be many patients on ventilators who will suit [this] treatment," Landray told The BMJ, adding that his team spent a week making sure the results were robust. They then shared their findings with "senior leaders in international healthcare," who made it clear that the group had to take action.

"We had a choice: do we wait for full publication or make information available to the world to make their own decision? If we hadn't released the [results], people would have said we sat on it," he says. covid-19. 


\section{Trial by design}

Landray emphasises that to put the trial in context it's important to cast our minds back to March and the sense of impending doom felt by clinicians in the UK.

"Covid-19 is a disease that affects huge numbers and where case fatality is high," he says. "More than one in four patients in hospital die, and of those admitted to intensive care units the prospect is worse. Back in early March none of us were sure if we were going to run out of ventilators or healthcare staff. Doctors in Italy were using words such as war zone.” The priority, he says, was to find a treatment that would reduce deaths-and ideally one where doctors could access a few thousand courses rapidly.

As the pandemic broke, the World Health Organization and the European Medicines Agency emphasised the need for large randomised trials with a control group rather than many small and inconclusive studies, as had come out of previous epidemics. 4

Landray and his colleagues wanted to embed research into standard clinical care and took inspiration from the large simple trials of the 1980s, in particular the International Studies of Infarct Survival (ISIS), randomised controlled trials of treatments for acute myocardial infarction. "It had to be easy for the clinician on the ground, in PPE and in a pressurised situation, and a minimal burden for the patient.," says Landray. "Many academic and commercial trials have accumulated so much extra baggage over the years, such as long case report forms and 10 page patient consent forms.”

To Ray Sheridan, a consultant physician at the Royal Devon and Exeter NHS Foundation Trust, which recruited 49 patients to RECOVERY, it seemed as though the NHS was "turning the engines on big time."

"You had 176 hospitals, and you could see the recruitment numbers going up astonishingly," he says. "There was an unprecedented level of interest. I'd say 95\% of patients were delighted there was a trial going on in their local hospital and felt like they were 'doing their bit' by being involved."

The mammoth task of mounting a large scale trial amid the first major pandemic in 100 years was accomplished in record time. Landray and fellow chief investigator Peter Horby drafted the protocol on $10 \mathrm{March}$, and the results for the dexamethasone arm were announced just 98 days later, after more than 11000 patients had been enrolled into the trial.

Patients enrolled in the open label RECOVERY trial are randomised to standard care or to one of six treatment arms: hydroxychloroquine (now ended), dexamethasone (also ended), lopinavir-ritonavir, azithromycin, convalescent plasma, and, in a second randomisation for patients who deteriorate, the anti-inflammatory drug tocilizumab.

For Sheridan, the trial meant that clinicians didn't have to make snap decisions on emerging treatments. There was a lot pressure to use hydroxychloroquine and azithromycin in combination, partly because there was emerging evidence from France, he says. "But it was non-randomised, and many of those patients weren't on oxygen, and a lot weren't in hospital for very long." Sheridan thought these were nothing more than pilot data, yet the study had begun to inform decision making about the pandemic.

"The RECOVERY trial stopped patients getting these treatments, and this was a relief," says Sheridan, "Otherwise, a lot of patients would be given steroids by some consultants, hydroxychloroquine by others, and ultimately we wouldn't be learning anything."
But for clinical pharmacologist John Warren, formerly of the MHRA, the RECOVERY approach to drug selection was too random. He says the approach seems "like a roulette wheel-here's an antiviral, let's try it."

Landray says the trial's choice of drugs was influenced by the New and Emerging Respiratory Virus Threats Advisory Group (NERVTAG), an expert committee of the Department of Health, and a WHO prioritisation process that took place in early 2020. The selection was ultimately governed by four principles: Is there a reason to believe the drug will work (for example, on the basis of laboratory test results or of experience from other viral infections)? Is the safety profile understood? Is the drug available in enough quantities to allow it to be tested in a trial of several thousand people? And, if the treatment is successful, can it be rapidly scaled up?

Other commentators have questioned the absence of the promising antiviral remdesivir in RECOVERY. The drug is currently being given to selected patients through the government's Early Access to Medicines Scheme after early trial data showed that it shortened time to recovery. ${ }^{5}$ The $B M J$ understands the lack of a remdesivir arm is because its manufacturer, Gilead, said it could offer only a limited supply of the drug and the investigators turned it down.

To this Landray would comment only that "we just couldn't get the supply we needed." But he added, "This is a global effort, and SOLIDARITY [a large multi-country WHO trial] is studying remdesivir in sufficient quantities. We don't want to repeat their effort. This is not competitive, and there was a reason to say, 'This is being done elsewhere, let's leave them to it."”

\section{Competition for patients}

At the start of the pandemic researchers were told to halt all non-covid-19 research and focus efforts on potential covid treatments, Landray says. The National Institute for Health Research (NIHR) says that there is a highly expedited process to approve applications and that a great many have been submitted. Researchers report that it can take weeks or months to get a decision. "The process is centralised and bureaucratic," says Beverley Hunt, medical director of the charity Thrombosis UK and a practising clinician. "And we don't know who is reviewing these proposals. There is huge frustration felt by many academics."

Hunt was, like others treating covid-19 patients with pneumonia, seeing high rates of hospital associated thromboembolism and other forms of thrombosis and thought that a good trial was urgently needed to compare different doses of anticoagulants. A consortium of UK thrombosis experts submitted a research proposal to the NIHR in April but were told it was a low priority area.

Hunt told The BMJ, "The problem was they had a preconceived view of what works and only wanted to look at antivirals and anti-inflammatories, and the problem of thrombosis was not obvious at the start of the pandemic in the UK." Eventually, after much discussion, an anticoagulation arm was added to the REMAP-CAP trial (involving patients with community acquired pneumonia being treated in intensive care).

The NIHR says that since March it has prioritised 48 public health research studies, out of more than 400 that have been assessed. 6

In contrast, RECOVERY gained momentum during the spring. For Landray, an important difference between RECOVERY and many other studies is when the study began: at the start of the pandemic, not in June, when patient numbers were falling off. The trial was aided by what Landray calls "fabulous" efforts by the NIHR's clinical research networks to cut red tape. Meanwhile, academics involved 
in smaller trials were getting frustrated at what they saw as an overtly bureaucratic process to give the green light to vital research.

James Galloway, a consultant rheumatologist at King's College Hospital in London and an investigator on a smaller trial called TACTIC, is impressed by the speed at which RECOVERY was set up: "Basically, hospitals could quickly switch on and deliver it." However, he has concerns about the sample size calculation, essentially seeking as many patients as possible. "For example, did we really need 1800 patients in the hydroxychloroquine arm to find out it didn't work? Could we have found this out sooner? That's 1800 patients who didn't go into other RECOVERY trial arms or even different studies." (He acknowledges that he has a personal bias as an investigator on a different trial.)

Landray says it is important to remember that RECOVERY is a "platform trial," looking at several drugs at the same time. "We knew we needed compelling evidence that a drug worked or didn't work, and so it was necessary that the trial was sufficiently powered. In the context of a disease that kills tens of thousands of people, a reduction in mortality of one fifth would have major

implications-for example, it is the difference between 20 ooo deaths and 16000 deaths. That is 4000 deaths prevented."

He adds, "Covid-19 is not a rare disease, so the overall impact of such modest treatment effects is massive. To be able to detect or dismiss such an effect requires a large trial. For example, a study with 2000 patients in the active arm and 4000 in the control arm would give $90 \%$ power at $\mathrm{P}=0.01$ to detect a risk reduction of about one fifth."

The trial is so large that it recruited around $15 \%$ of all patients with covid-19 in UK hospitals, though Landray points out that of course this means $85 \%$ of such patients are not in the trial. Some patients would not have wanted to enter a trial, others may not have been approached, and some may not have been suitable for some reason. At some hospitals up to $80 \%$ of covid patients were recruited, while in others it was as low as $3 \%$.

\section{The trial's legacy}

For many involved in RECOVERY, the longer term issue is how the trial can reset the way clinical trials are conducted in the future. Landray says, "How can we build on the involvement of patients and clinicians and the timely access to relevant data? We now need to apply the lessons from this approach to other major health challenges such as heart disease, cancer, arthritis, and mental health."

Ray Sheridan is keen to keep up the momentum. "We have seen things done in a really streamlined, efficient way, and people really want to hold on to that. We liked the fact that it was really pragmatic, so the moment you know a drug is not working it gets dropped and then other drugs can get added. It also shows that you can use the whole of the NHS rather than just the main academic centres.

"You have a whole lot of people in hospitals who are not usually involved in research on a day to day basis who really want to carry on."

\section{BoX 1: RECOVERY trial timeline}

- 10 March-Trial protocol is drafted

- 11 March-WHO declares the covid-19 outbreak a pandemic

- 19 March-First RECOVERY patient is recruited

- 23 March-UK goes into lockdown

- 14 May-10 oooth RECOVERY patient is recruited
- 5 June-Alerted by its data monitoring committee, the trial halts the hydroxychloroquine arm, concluding that the drug has no clinical benefit for patients in hospital with covid-19

- 9 June-Trial's statistical analysis plan is published

- 16 June-Results of the dexamethasone arm announced by press release

- 22 June-Dexamethasone preprint published on medRxiv

- 29 June-Results of lopinavir-ritonavir arm indicate no clinical benefit in hospital patients with covid-19

\section{Box 2: The trial's hydroxychloroquine dosage}

The high doses of hydroxychloroquine used in RECOVERY-80o mg at o and 6 hours followed by $400 \mathrm{mg}$ at 12 hours and then every 12 hours for up to nine additional days-have raised concern among experts.

David Jayne, professor of clinical autoimmunity at Cambridge University, said that current recommended doses for rheumatologic disease are typically $300-400 \mathrm{mg} /$ day and that the maximum dose for malaria has been $800 \mathrm{mg}$ in the first 24 hours. "The reasons behind the dose selection in the RECOVERY trial are unclear," he says. "Hydroxychloroquine overdose is associated with cardiovascular, neurological, and other toxicities, occurring with doses over $1500 \mathrm{mg}$, and higher doses are associated with fatality." He is concerned that hydroxychloroquine toxicity may have contributed to the adverse outcomes and that conclusions based on these results may be unreliable.

Martin Landray, RECOVERY's deputy chief investigator, says, "We did not choose these doses by accident. The dose comes from modelling by Nick White, professor of tropical medicine at the University of Oxford, and his team, who have extensive experience with this drug. They developed detailed pharmacokinetic models, considering the best way in which to rapidly achieve drug levels that might be high enough to kill the virus but not so high as to trigger toxicity. Their work has recently been published as a preprint on medRxiv."

\section{Box 3: Leading the research on the ward}

When it comes to conducting urgent lifesaving research, watching videos on the internet isn't what normally springs to mind.

"When you do research trials, certainly for industry ones, there's masses of e-learning to do, and it is really offputting," says Ray Sheridan, consultant physician at the Royal Devon and Exeter trust, where 49 patients were recruited to RECOVERY. "A lot of it is really pointless, but RECOVERY's was very doable and just had a 5-10 minute video to watch for each section."

Sheridan says the protocol was very straightforward. "We set up $24 / 7$ access to research nurses, but often enough I did the randomisation myself, it was so straightforward. We used web based data entry forms that took you a matter of minutes to enter someone into the trial."

All patients had to be have been admitted to hospital to qualify, but participation was not limited to just those in intensive care. "In a lot of trials, if you are 90 and have dementia you don't go into routine trials, whereas these were all comers," says Sheridan.

Staff had plenty of time to discuss the trial with potential participants, despite the emergency nature of their infection. "There was never any pressure on patients to go into the trial, and often they wanted to talk to their families as well," says Sheridan, who is also involved in

neurodegenerative disorder trials, where "people want a lot more time to think about it." For those there is a $20-30 \%$ uptake, but with RECOVERY almost everyone they spoke to wanted to do it. Sheridan says, "We were absolutely clear that we didn't know whether these drugs worked and that this is the only way we can find out if they do. This was quite brave of patients, as one of the arms was standard care, but this didn't put them off."

Sheridan says his clinicians felt in complete control, having had long discussions about the pros and cons of the various drugs tested before finally deciding to help test all of them. "There were a lot of reservations about the steroid arm, and some of the data coming through suggested 
the HIV drug wasn't working, based on one or two studies in China, although those patients were recruited after 11 days of being in hospital whereas we were looking from day 1 . The fact that we went into all arms, open without the prejudices, felt good to me. If we'd been really clear that we didn't like one arm, that would have been uncomfortable." Sheridan adds, "We went into this pandemic with a real sense of doom and gloom. We were really expecting to be like Italy, but we felt we turned the wards into a positive place. Patients were coming in terrified. But we were running research trials and were offering our patients the best possible options, and that helped overall."

\section{BOX 4: Transparency in ACCORD}

RECOVERY isn't the only covid-19 trial or research platform facing questions about transparency.

On 29 April England's health and social care secretary, Matt Hancock, announced the Accelerating Covid-19 Research and Development (ACCORD) programme, which will look at potential drugs that could be fast tracked through early stage clinical trials. ${ }^{7}$ If they show promise they would then be fed into large scale studies such as RECOVERY.

The drugs so far confirmed are MEDI3506 (an anti-IL-33 monoclonal antibody), zilucoplan (a complement $\mathrm{C}_{5}$ inhibitor that could block severe inflammatory responses), bemcentinib (an AXL inhibitor, with early data showing it can reduce viral infection and lung inflammation and block the SARS-CoV-2 spike protein), and acalabrutinib (a Bruton's tyrosine kinase (BTK) inhibitor developed for severe lung inflammation).

The decisions on which drugs are included in ACCORD are made by a "therapeutics taskforce," but its membership is not listed anywhere accessible, and there are no published terms of reference or published minutes. A spokesperson for the Department of Health and Social Care said that the taskforce is being led by the deputy chief medical officer for England, Jonathan Van-Tam, but would not confirm any other members. The spokesperson said, "The taskforce does not choose the drugs that go to trial as these are recommended by the prioritisation panel. The panel includes over 20 contributors, including frontline NHS clinicians, academics with expertise in covid-19 disease mechanisms, and relevant expertise from the life sciences sector. The current membership of the taskforce is under review, and we will publish the full list, alongside the members of the prioritisation panel, when this is completed."

ACCORD reports to the business secretary, Alok Sharma. The DHSC's website states that it is a partnership between the Government Scientific Office, the NIHR, the clinical research company IQVIA, and the drug company AstraZeneca. Of the five drugs publicly released in the current ACCORD trial, two are AstraZeneca compounds: interleukin 33 (IL-33) and acalabrutinib.

There are concerns that the taskforce is influenced by the industry and acting as the conduit for political direction of clinical research. The clinical pharmacologist John Warren told The BMJ that the taskforce should include a medicinal chemist, pharmacologist, pharmacokineticist, virologist, immunologist, and toxicologist.

\section{Box 5: Main covid-19 drug trials in UK}

- ACCORD-A clinical trial platform to assess candidate agents that runs in alliance with a national collaboration of phase 2 drug development platforms (www.accord-trial.org)

- CATALYST-A trial to test the anti-inflammatory drug infliximab, currently used to treat rheumatoid arthritis and inflammatory bowel syndrome

- PRINCIPLE-A platform trial of interventions in older people carried out in primary care (www.phctrials.ox.ac.uk/principle-trial)

- RECOVERY-A platform trial evaluating existing or new drugs in patients being treated in hospital (www.recoverytrial.net)

- REMAP-CAP-A platform trial for critically ill patients (www.remapcap.org)
- TACTIC-A trial to test baricitinib, a drug used to treat rheumatoid arthritis, and the monoclonal antibody ravulizumab (https://cctu.org.uk/portfolio/COVID-19/TACTIC)

\section{Competing interests: None declared.}

Commissioning and peer review: Commissioned, not peer reviewed.

1 Mahase E. Covid-19: Low dose steroid cuts death in ventilated patients by one third, trial finds. BMJ 2020;369:m2422. doi: 10.1136/bmj.m2422 pmid: 32546467

2 Mahase E. Hydroxychloroquine for covid-19: the end of the line?BMJ2020;369:m2378. doi: 10.1136/bmj.m2378 pmid: 32540958

3 Torjesen I. Covid-19: Hydroxychloroquine does not benefit hospitalised patients, UK trial finds. BMJ 2020;369:m2263. doi: 10.1136/bmj.m2263 pmid: 32513810

4 European Medicines Agency. A call to pool EU research resources into large-scale, multi-centre multi-arm clinical trials against covid-19.16 Mar 2020. https://www.ema.europa.eu/en/documents/other/call-pool-eu-research-resources-large-scale-multi-centre-multi-arm-clinical-trialsagainst-covid-19_en.pdf.

$5 \quad$ Kmietowicz Z. Covid-19: Selected NHS patients will be treated with remdesivir. BMJ 2020;369:m2097. doi: 10.1136/bmj.m2097 pmid: 32457029

6 Watson JA, Tarning J, Hoglund RM, et al. Concentration-dependent mortality of chloroquine in overdose [preprint]. medRxiv 2020.04.24.20078303. http://medrxiv.org/lookup/doi/10.1101/2020.04.24.20078303.

7 Department of Health and Social Care. Covid-19 treatments could be fast-tracked through new national clinical trial initiative. 29 Apr 2020. https://www.gov.uk/government/news/covid-19. treatments-could-be-fast-tracked-through-new-national-clinical-trial-initiative. 